\title{
ThermoData Engine (TDE): Software Implementation of the Dynamic Data Evaluation Concept. 2. Equations of State on Demand and Dynamic Updates over the Web
}

\author{
Vladimir Diky, Chris D. Muzny, Eric W. Lemmon, Robert D. Chirico, and Michael Frenkel* \\ Physical and Chemical Properties Division, National Institute of Standards and Technology, \\ Boulder, Colorado 80305-3328
}

Received February 21, 2007

\begin{abstract}
ThermoData Engine (TDE) is the first full-scale software implementation of the dynamic data evaluation concept, as reported recently in this journal. The present paper describes two major software enhancements to TDE: (1) generation of equation of state (EOS) representations on demand and (2) establishment of a dynamically updated experimental data resource for use in the critical evaluation process. Four EOS formulations have been implemented in TDE for on-demand evaluation: the volume translated PengRobinson, modified Sanchez-Lacombe, PC-SAFT, and Span Wagner EOS. The equations are fully described with their general application. The class structure of the program is described with particular emphasis on special features required to implement an equation, such as an EOS, that represents multiple properties simultaneously. Full implementation of the dynamic data evaluation concept requires that evaluations be based on an up-to-date "body of knowledge" or, in the case of TDE, an up-to-date collection of experimental results. A method to provide updates through the World Wide Web is described that meets the challenges of maintenance of data integrity with full traceability. Directions for future enhancements are outlined.
\end{abstract}

\section{INTRODUCTION}

As discussed in the first paper of this series, ${ }^{1}$ NIST ThermoData Engine (TDE) ${ }^{2}$ software represents the first fullscale implementation of the dynamic data evaluation concept for thermodynamic and thermophysical properties., ${ }^{3,4}$ This concept requires large electronic databases capable of storing essentially all relevant experimental data known to date with detailed descriptions of metadata and uncertainties. The combination of these electronic databases with expert-system software, designed to automatically generate recommended data based on available experimental and predicted data, leads to the ability to produce critically evaluated data dynamically or 'to order'.

Critical evaluation of thermodynamic and thermophysical property data can be performed based on one of three general methods: (1) single-property data analysis; (2) simultaneous analysis of multiple properties with enforced mutual consistency; or (3) through application of an equation-of-state (EOS). ${ }^{5,6}$ The first two approaches were implemented previously in TDE for dynamic data evaluation..$^{1,2}$ Implementation of dynamically evaluated EOS representations is addressed in the present paper. At this time, all discussions are restricted to pure molecular compounds only.

Implementation of the EOS technology allows, in principle, evaluation of all thermodynamic properties of the system simultaneously. In general, an EOS establishes a relationship between the thermodynamic variables of the system, temperature $T$, volume $V$, and pressure $p$. For one mole of pure substance, the equation of state can generally be expressed as

$$
p V=R T+f(T, p)
$$

where $R$ is the gas constant.
In the case of an ideal gas, where interactions between molecules are absent, the function $f(T, p)$ is equal to zero. In practice, this term is rarely negligible and has been calculated with a wide variety of numerical approximations leading to the development of hundreds of empirical EOS formulations during the last century. ${ }^{7}$ More precise equations of state often contain numerous parameters for characterizing $f(T, p)$. These equations can be deployed only if the compound of interest has been studied adequately to allow determination of the fitting parameters. Data of high quality and broad range are often required for a high-precision EOS. Some less precise EOS formulations containing only a few parameters can be deployed for a large variety of "data scenarios", including compounds for which data are sparse. In either event, until now, EOS technology has been deployed in only static data evaluations. ${ }^{8,9}$ EOS deployment in dynamic data evaluation is particularly challenging for complex, highprecision equations, due to mathematical complexities, the sensitivity of results to data quality, the necessity to meet special validity criteria, and the need to function without human intervention. Dynamic evaluation provides new opportunities for generating EOS representations for a wide variety of chemical species. A key aspect is the establishment of criteria for deployment of a specific type of EOS based on a particular data scenario, i.e., the data quality and extent.

Full implementation of the dynamic data evaluation concept requires that evaluations be based on an up-to-date "body of knowledge".,3 To meet this requirement, a system must be established to provide regular updates of available experimental thermodynamic property data. The World Wide Web can provide a path for such updates, but important challenges include maintenance of data integrity and full traceability. 
The present paper describes a software implementation ${ }^{10}$ of the dynamic data evaluation concept for both principal areas outlined above: (1) generation of EOS representations on-demand and (2) establishment of a dynamically updated experimental data resource for use in the critical evaluation process.

In order to adequately represent a range of data scenarios, four different equations of state of various complexity and precision were selected for implementation: Peng-Robinson, ${ }^{11}$ PC-SAFT, ${ }^{12}$ Sanchez-Lacombe, ${ }^{13,14}$ and fundamental equations based on the Helmholtz energy. ${ }^{15,16}$ A dynamically updated TDE-SOURCE ${ }^{1,17}$ data storage facility was developed, in part, using the multitier Web-Oracle dissemination system $^{18}$ recently established in development of the NIST Ionic Liquids Database, ILThermo. ${ }^{19}$

\section{SELECTED EQUATIONS OF STATE}

Equations of state are the underlying basis for the calculation of thermodynamic properties for any fluid. Often, the properties of a pure fluid are represented by a variety of equations; for example, the vapor pressure can be represented by a Wagner type equation and the liquid density with a Tait type equation (cf. ref 20). Multiple equations may be used to span wide ranges of density, where one equation is often used for the liquid, one for the vapor, and possibly a third for supercritical conditions. The critical region may be represented by scaling law equations. The application of multiple equations often results in discontinuities at the borders between equations and inconsistencies between mathematically related properties, such as the vapor pressure and the enthalpy of vaporization.

Fundamental EOS Formulations based on the Helmholtz Energy. These shortcomings can be avoided by using a single equation of state to represent all of the thermodynamic properties for the fluid of interest. Most modern, widerange, high-precision equations of state for pure fluid properties are fundamental equations explicit in the Helmholtz energy as a function of density and temperature. The advantage of using fundamental equations of state is that all single-phase thermodynamic properties can be calculated as derivatives of the Helmholtz energy. For example

$$
p=\rho^{2}\left(\frac{\partial a}{\partial \rho}\right)_{T}
$$

where $p$ is the pressure, $\rho$ is the density, and $a$ is the specific Helmholtz energy. Equations for other properties such as the speed of sound are more complex but still require only derivatives of the Helmholtz energy. The location of the saturation boundaries requires an iterative solution of the physical constraints on saturation (the so-called Maxwell criterion that pressures and Gibbs energies are equal at constant temperature during phase changes). Such computational complexities are readily justified, as the result is a fully consistent set of property values.

Equations of state explicit in the Helmholtz energy as the fundamental property with independent variables of density and temperature can be expressed as

$$
a(\rho, T)=a^{0}(\rho, T)+a^{\mathrm{r}}(\rho, T)
$$

where $a^{0}(\rho, T)$ is the ideal gas contribution to the Helmholtz energy and $a^{\mathrm{r}}(\rho, T)$ is the residual Helmholtz energy, which corresponds to the influence of intermolecular forces. The Helmholtz energy of the ideal gas can be formulated in terms of the ideal gas heat capacity $c_{p}{ }^{0}$. Equations for $a^{0}(\rho, T)$ are developed by fitting experimental data for either heat capacities at low pressures, speeds of sound in the gas phase, or values derived from spectroscopic information and the methods of statistical mechanics.

Unlike the case of an ideal gas, the real fluid behavior is often described with empirical models that are only loosely supported by theoretical considerations. Although it is possible to extract values such as second and third virial coefficients from the fundamental equation, the terms in the equation are empirical, and any functional connection to theory is not entirely justified. The coefficients of the equation depend on the experimental data for the fitted fluid.

A common functional form for Helmholtz energy equations of state 21,22 is

$$
a^{\mathrm{r}}(\delta, \tau)=\Sigma N_{k} \delta^{d_{k}} \tau^{t_{k}}+\Sigma N_{k} \delta^{d_{k}} \tau^{t_{k}} \exp \left(-\delta^{l_{k}}\right)
$$

where $\delta$ is the reduced density $\left(\delta=\rho / \rho_{\mathrm{c}}\right.$ ) and $\tau$ is the inverse reduced temperature $\left(\tau=T_{\mathrm{c}} / T\right)$. Each summation typically contains 4-20 terms, where the index $k$ points to each individual term. The values of $d_{k}, t_{k}$, and $l_{k}$ are restricted so that the $t_{k}$ are generally expected to be greater than zero, and $d_{k}$ and $l_{k}$ are integers greater than zero. Further information on equations of state and their applications to several fluids is given by Lemmon and Jacobsen ${ }^{21}$ and Lemmon and Span. ${ }^{22}$

Span-Wagner EOS Formulations. The fundamental equations of state used in TDE are based on the work of Span and Wagner. ${ }^{16}$ Their work describes the development of two 12-term equations with fixed functional forms: one for nonpolar or slightly polar substances and one for polar fluids. Data sets of high quality were used to develop the functional forms and assess the quality of the resulting data representations. Although the new technical equations suffer from a slight loss in representation accuracy for some compounds with very extensive high-quality data, their shorter forms allow for computations that are as much as ten times faster, depending on the number of terms in the other equations and on the use of special constraints for fitting in the critical region.

The technical equations of Span and Wagner ${ }^{16}$ were developed with keen insight into the proper behavior of an equation of state. They extrapolate properly at low temperatures (as demonstrated by the curvature of the isobaric and isochoric heat capacities, and the speed of sound) and at high temperatures (for example, along the "ideal curve" with compressibility factor $Z=1$ ). In addition, the number of terms in the eqs (12 each) was kept to a minimum, which decreases the extent of correlation among terms and overfitting. Although the smaller number of terms decreases the flexibility of the equation, and thus its ability to accurately represent a fluid's properties, its rigid shape allows greater application to substances with limited data. In this way, the fitted equation becomes a reliable tool to fill in gaps in the thermodynamic surface. These fixed functional forms are ideal for use in TDE to represent fluids that are often characterized by limited data sets; however, they are not 
recommended for representation of highly polar molecules, such as acids and alcohols.

Other EOS Formulations Implemented in TDE. Three EOS formulations that are used in the engineering community are (a) the volume-translated Peng-Robinson equation ${ }^{11}$ with a substance-dependent constant volume-translation term (described later), (b) the PC-SAFT equation, ${ }^{12}$ and (c) the volume-translated (commonly referred to as the "modified") Sanchez-Lacombe equation. ${ }^{13,14}$ Although none of these equations approach the precision possible with the fundamental EOS formulations described above, they do have numerous engineering applications, particularly when few or only low-quality experimental data are available. These equations are implemented in TDE, and their formulations are described in the following paragraphs.

The Volume-Translated Peng-Robinson EOS. The Peng-Robinson equation is

$$
Z=V(V-b)^{-1}-\Theta V /\left\{(R T)\left(V^{2}+\delta V+\epsilon\right)\right\}^{-1}
$$

where $Z$ is the compressibility factor $(p V / R T), p$ is the pressure, $V$ is the molar volume, $R$ is the gas constant, and $T$ is the temperature. The quantity $\Theta$ is defined based on the following expressions

$$
\Theta=a \alpha
$$

where

$$
\begin{gathered}
\alpha=\left\{\left(-0.26992 \omega^{2}+1.54226 \omega+0.37464\right)\left(1-T_{\mathrm{r}}^{0.5}\right)+\right. \\
1\}^{2}(7) \\
a=0.45724\left(R T_{\mathrm{c}}\right)^{2} / p_{\mathrm{c}}
\end{gathered}
$$

and

$$
T_{\mathrm{r}}=T / T_{\mathrm{c}}
$$

where $\omega$ is the acentric factor, $T_{\mathrm{c}}$ is the critical temperature, and $p_{\mathrm{c}}$ is the critical pressure. Finally, the equation quantities $b, \delta$, and $\epsilon$ are defined as follows:

$$
\begin{gathered}
b=0.0778 R T_{\mathrm{c}} / p_{\mathrm{c}} \\
\delta=2 b \\
\epsilon=-b^{2}
\end{gathered}
$$

The dimensionless residual Helmholtz energy $a^{r}$ for the Peng-Robinson equation was obtained by integration

$$
a^{r}=\left(A-A^{0}\right) / R T=\int(Z-1) \rho^{-1} \mathrm{~d} \rho
$$

where $A$ is the Helmholtz energy for the real-fluid, $A^{0}$ is the ideal-gas Helmholtz energy at the same temperature and molar density, and $\rho$ is the molar density.

As described later in the section The EOS Fitting Process, the critical temperature, critical pressure, and acentric factor are fixed through an initial critical evaluation by TDE prior to the EOS implementation. Consequently, there is only one adjustable parameter in fitting the Peng-Robinson equation, the volume-translation term $v_{\text {tr }}$. The initial value is calculated by the formula ${ }^{23}$

$$
\begin{aligned}
v_{\mathrm{tr}}=\left(R T_{\mathrm{c}} / p_{\mathrm{c}}\right)(-0.014471+0.067498 \omega- \\
\left.0.084852 \omega^{2}+0.067287 \omega^{3}-0.017366 \omega^{4}\right)
\end{aligned}
$$

"Volume translation" 24 means that the volume $V$, calculated with the original Peng-Robinson equation (eq 5, without volume translation) as a function of temperature and pressure in the single phase region or as a function of temperature on the vapor-liquid saturation line, is shifted by the volume-translation term

$$
V_{\mathrm{t}}=V-v_{\mathrm{tr}}
$$

where $V$ is the volume (of eq 5) that is thermodynamically consistent with all other properties, $v_{\text {tr }}$ is the volumetranslation term, and $V_{\mathrm{t}}$ is the adjusted volume. It is important to note that $V_{\mathrm{t}}$ is not consistent with all other properties derived with the volume-translated equation. However, this adjusted volume is a better approximation to the observed volume that can be measured experimentally. As follows from this, the volume-translation term affects only singlephase and saturated liquid and gas-phase densities and, in theory, can be refined by fitting any combination of experimental values for these properties. This option as well as the particular state-variable region to fit can be selected by the user. The volume-translated Peng-Robinson equation generally provides a representation that often lies well outside the experimental uncertainty, but calculations are very fast and are widely used in industrial applications.

The Modified Sanchez-Lacombe EOS. The SanchezLacombe equation is another EOS formulation that includes volume translation. Its mathematical form (without volume translation) and application was described by Koak and Heidemann. ${ }^{13}$ The equation is defined in terms of the compressibility factor $Z$ as follows

$$
Z=p V /(R T)
$$

where $p$ is expressed as

$p=\left(-\delta^{2} \epsilon \nu / V^{2}\right)-R T[(\delta-1) / V+\ln \{1-\delta v / V\} / v]$

with the equation parameters $\delta, \epsilon$, and $v$ defined as follows

$$
\begin{gathered}
\delta=5.1178+13.5698 \omega+5.9404 \omega^{2}-1.2952 \omega^{3} \\
\epsilon=R T_{\mathrm{c}}\left(1+\delta^{0.5}\right)^{2} / 2 \delta \\
v=\left(R T_{\mathrm{c}} / p_{\mathrm{c}}\right)\left[\ln \left(\delta^{-0.5}+1\right)-\left\{\left(\delta^{0.5}-0.5\right) / \delta\right\}\right]
\end{gathered}
$$

All other symbols $\left(V, R, T, \omega, T_{\mathrm{c}}\right.$, and $\left.p_{\mathrm{c}}\right)$ are as defined for the Peng-Robinson equation.

Volume translation for the Sanchez-Lacombe EOS was proposed by Krenz. ${ }^{14}$ The volume-translation term $v_{\text {tr }}$ is expressed as

$$
v_{\mathrm{tr}}=(3.297 \delta-0.1092 M) / 1000
$$

where $M$ is the molar mass in $\mathrm{g} \cdot \mathrm{mol}^{-1}$, and $v_{\text {tr }}$ is in units of $\mathrm{dm}^{3} \cdot \mathrm{mol}^{-1}$.

The PC-SAFT EOS. The PC SAFT (Perturbed-Chain Statistical Associating Fluid Theory) equation is based on intermolecular potential theory and is not a volume-translated equation. It is implemented in TDE in the formulation described by Gross and Sadowski, ${ }^{12}$ where the primary 
Table 1. General Characteristics of the EOS Formulations in the ThermoData Engine (TDE) Software

\begin{tabular}{|c|c|c|c|c|c|}
\hline $\begin{array}{l}\text { equation of state } \\
\text { (EOS) }\end{array}$ & $\begin{array}{l}\text { required } \\
\text { properties }\end{array}$ & $\begin{array}{l}\text { adjustable } \\
\text { parameters }\end{array}$ & $\begin{array}{l}\text { thermodynamic } \\
\text { consistency }\end{array}$ & $\begin{array}{c}\text { data extent } \\
\text { for optimal } \\
\text { representation }\end{array}$ & $\begin{array}{c}\text { precision } \\
\text { of } \\
\text { representation }\end{array}$ \\
\hline volume-translated & $T_{\mathrm{c}}, p_{\mathrm{c}}, \omega$ & 1 & no & low & low \\
\hline $\begin{array}{l}\text { Peng-Robinson } \\
\text { modified } \\
\text { Sanchez-Lacombe }\end{array}$ & $T_{\mathcal{c}}, p_{\mathrm{c}}, \omega$ & 1 & no & low & low \\
\hline PC-SAFT & none & 3 & yes & medium & medium \\
\hline Span-Wagner & $T_{\mathrm{c}}, \rho_{\mathrm{c}}$ & 12 & yes & high & high \\
\hline
\end{tabular}

quantity calculated is the dimensionless residual Helmholtz energy $a^{r}$. (Gross and Sadowski later published a formulation that included an association term, ${ }^{25}$ but this is not implemented in the version of TDE described here.) For the PCSAFT equation, $a^{r}$ is calculated with three adjustable parameters, $m, \sigma$, and $\epsilon$, and two constant arrays, $a$ and $b .^{12}$ The mathematical formulation of the PC-SAFT EOS is complex. For the purpose of completeness, the formulation is included as an Appendix to this article. Due to the complexity of the mathematics, the derivatives of the residual Helmholtz energy with respect to temperature and density that are necessary for the calculation of thermodynamic properties were derived with a symbolic mathematics package $^{26}$ and then translated to the $\mathrm{C}++$ programming language.

The PC-SAFT EOS can describe vapor pressure and saturation density with good precision but commonly overestimates the critical temperature $T_{\mathrm{c}}$. Experimental vapor pressures are well represented even to the experimental $T_{\mathrm{c}}$; however, saturation densities near $T_{\mathrm{c}}$ are often erroneous because of the overestimation of $T_{\mathrm{c}}$.

Summary. General characteristics of the four EOS formulations presently in TDE are summarized in Table 1. As can be seen in the table, the volume-translated PengRobinson EOS and modified Sanchez-Lacombe EOS have the same requirements and general expectations for quality of property representation. In comparison with the PengRobinson EOS (with the original $\alpha$ function, eq 7 , implemented in TDE 2.0), the modified Sanchez-Lacombe equation better describes vapor pressure but diverges from the saturation density for the liquid at high temperatures. Also, the extent of the volume-translation adjustment is greater for the modified Sanchez-Lacombe EOS than that for the volume-translated Peng-Robinson EOS. Consequently, parametrization for a restricted temperature range is suggested for a particular application for the volume-translated equations of state. While the volume-translation method works well in the variable range where the translation is made, a volume-translated EOS can give results that are worse than a simple cubic EOS under conditions away from where the translation parameters were established. Among the four equations provided, optimum equation selection is necessarily dependent on the target application and the data scenario for the target compound.

\section{CRITERIA FOR EOS DEPLOYMENT}

As noted, the equations of state chosen for inclusion in TDE are a very small subset of the plethora of published formulations. The selected equations were chosen, in part, to provide choices that would be serviceable for a broad variety of data scenarios ranging from extensive, high quality data, such as that available for the common hydrocarbon gases, to effectively none, such as for new or hypothetical compounds.

There are specific sets of properties that are necessary to define each EOS. For the volume-translated Peng-Robinson and modified Sanchez-Lacombe equations, only the critical temperature $T_{\mathrm{c}}$, critical pressure $p_{\mathrm{c}}$, and acentric factor $\omega$ are required. Technically, no constants are needed to define a PC-SAFT equation, but in practice, application is restricted to compounds with a defined critical temperature, either experimental or predicted by TDE.

Due to the greater number of adjustable parameters, the criteria for successful deployment of the Span-Wagner EOS are more complex and are shown as a flow diagram in Figure 1. If the available data allow definition of an equation (i.e., if the critical temperature $T_{\mathrm{c}}$ and critical density $\rho_{\mathrm{c}}$ are available), a series of additional criteria is used to provide warnings to the user during the EOS fitting process. If idealgas heat capacities are not available, the Span-Wagner equation cannot be deployed for energy related properties. Available validated ideal-gas heat capacities ${ }^{27}$ are stored in the TDE database. The ideal-gas heat capacities are necessary for EOS-based calculation of heat capacities and speeds of sound. Based on the available data, a prognosis for fitting success is made, and the user is warned in the case of a negative or doubtful prognosis (Figure 1). Warnings are provided for compounds with strong intermolecular association (e.g., water, alcohols, and acids) and compounds with normal boiling temperatures above $700 \mathrm{~K}$ (e.g., the branched hydrocarbon squalane with empirical formula $\mathrm{C}_{30} \mathrm{H}_{62}$ ). In our experience, properties for such compounds are not well described by the 12-parameter Span-Wagner EOS. (It is unclear whether this is due to a limitation of the EOS formulation or the quality of the available predicted and experimental property data.) The absence of experimental vapor pressures, saturated liquid densities, and saturated liquid heat capacities, also, gives a negative prognosis for fitting. In addition, the lack of single-phase liquid densities at pressures $p>200 \mathrm{kPa}$ limit the quality of a Span-Wagner EOS representation.

\section{THE EOS FITTING PROCESS}

In order to define an EOS, there are specific properties (e.g., the critical temperature, acentric factor, etc., depending upon the specific EOS) that must be evaluated prior to deployment of an EOS. The first paper in this series ${ }^{1}$ described in detail the dynamic data evaluation methods used in TDE for evaluation of such properties. Fitting an EOS within TDE involves two steps: initial evaluation to provide needed constants and parameters, followed by EOS fitting.

Interface options support the process of EOS deployment for a compound, including equation scaling, fitting, plotting, 


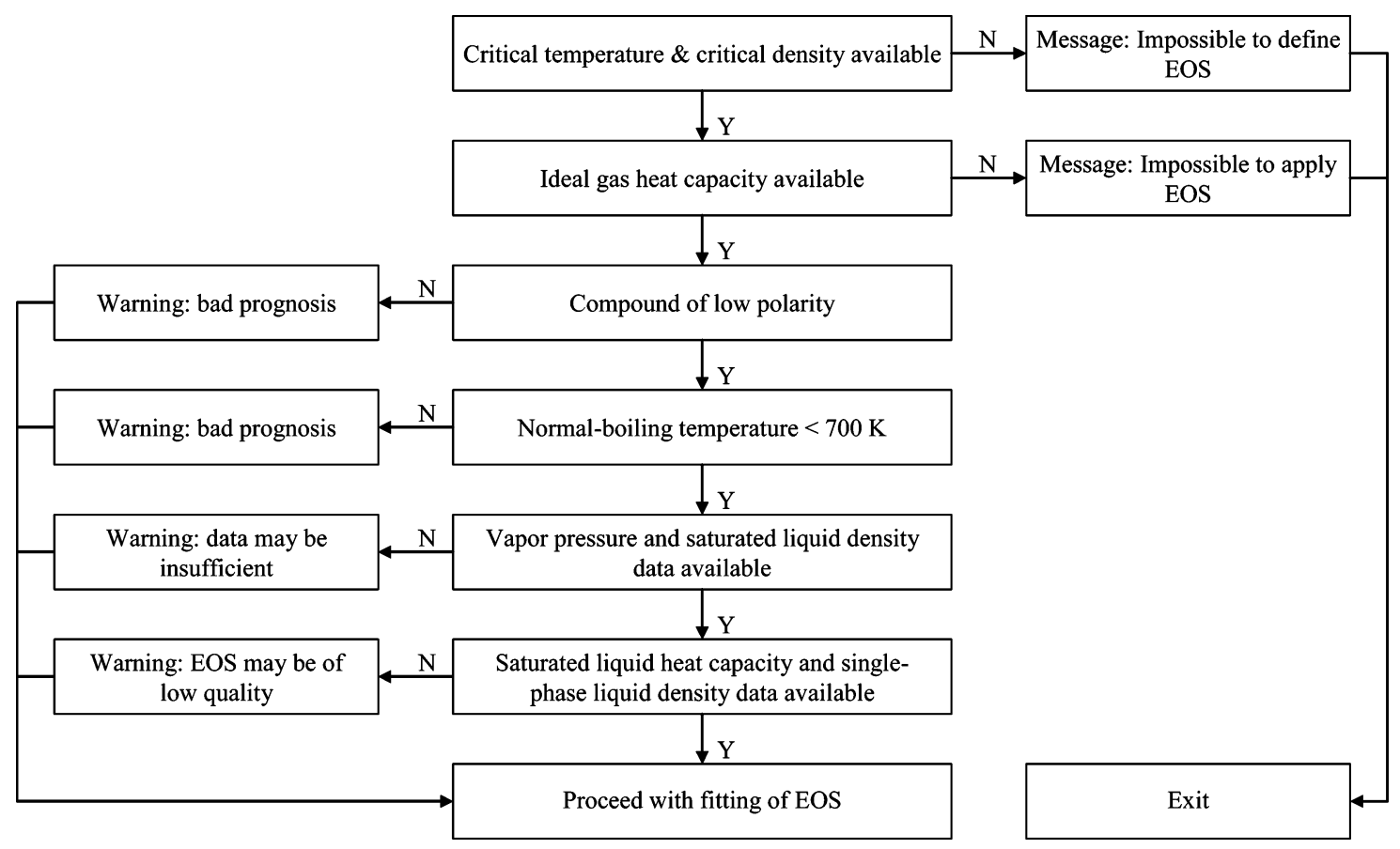

Figure 1. Algorithm for deployment criteria for the Span-Wagner equation of state.

\begin{tabular}{|c|c|c|c|c|c|c|c|c|c|c|}
\hline \multicolumn{10}{|c|}{ TिE ThermoData Engine - toluene } & \multirow[t]{2}{*}{ 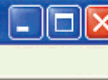 } \\
\hline \multicolumn{10}{|c|}{ Fille View Action EOS Updates Help } & \\
\hline \multirow{5}{*}{\multicolumn{2}{|c|}{ 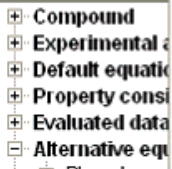 }} & RefProp EOS (Read-only) & \multicolumn{7}{|c|}{ 問 EOS Control Center: Span-Wagner } & $-\square x$ \\
\hline & & & Property & Weight Factor & Data Points & Relative Weight & Starting Error & Current Error & Adequacy & \\
\hline & & Span-Wagner(Non-polar) & \multirow{30}{*}{ 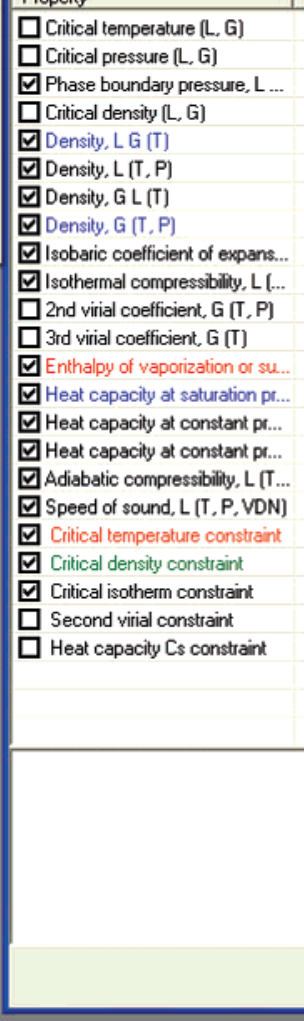 } & 1 & 21 & 97.1 & 0.000 & 0.000 & & \\
\hline & & Span-Wagner(Polar) & & 1 & 14 & 1.26 & 0.000 & 0.000 & & \\
\hline & & & & 1 & 815 & 181 & 277 & 277 & 0.339 & \\
\hline & 2. Phase bounc & Save EOS & & 1 & 6 & 0.0186 & 0.000 & 0.000 & & \\
\hline & Density & PC-SAFT & & 1 & 781 & $8.13 e+003$ & $6.22 \mathrm{e}+003$ & $6.22 e+003$ & 7.97 & \\
\hline & - Heat capacit & Modified Sanchez-Lacombe & & 1 & 917 & 743 & 382 & 382 & 0.416 & \\
\hline & Heat capacit & Translated Peng-Robinson & & 1 & 6 & 0.0383 & 5.79 & 5.79 & 0.965 & \\
\hline & Surface tensi & & & 1 & 134 & 14.3 & 934 & 934 & 6.97 & \\
\hline & Viscosity & EOS Control Center & & 1 & 24 & 0.00150 & 0.872 & 0.872 & 0.0363 & \\
\hline & Thermal cond & sctivity & & 1 & 20 & 0.00158 & 1.50 & 1.50 & 0.0749 & \\
\hline E & quations of st & & & 1 & 137 & 6.84 & 0.000 & 0.000 & & \\
\hline & Span-Wagner & & & 1 & 31 & 3.58 & 0.000 & 0.000 & & \\
\hline & Critical ter & perature, LG & & 1 & 35 & 19.2 & $1.21 \mathrm{e}+003$ & $1.21 \mathrm{e}+003$ & 34.6 & \\
\hline & Critical pre & ssure, LG & & 1 & 121 & 2.96 & 577 & 577 & 4.77 & \\
\hline & Phase bo & indary pressure, L G (T) & & 1 & 7 & 0.0157 & 0.809 & 0.809 & 0.116 & \\
\hline & Critical de & nsity, L G & & 1 & 3 & 0.142 & 0.875 & 0.875 & 0.292 & \\
\hline & Density, L & $G(T)$ & & 1 & 1 & 0.00918 & 0.0675 & 0.0675 & 0.0675 & \\
\hline & Density, L & (T,P) & & 1 & 0 & 0.000 & 0.000 & 0.000 & 1.00 & \\
\hline & Density, G & $\mathrm{L}(\mathrm{T})$ & & 1 & 1 & $3.35 e+003$ & 91.0 & 91.0 & 91.0 & \\
\hline & Density, G & {$[T, P]$} & & 1 & 1 & 7.53 & 1.37 & 1.37 & 1.37 & \\
\hline & Isobaric o & sefficient of expansion, L (T, F & & 1 & 1 & 0.000 & 0.000 & 0.000 & & \\
\hline & Isotherma & compressibility, L (T, P] & & 1 & 1 & 0.000 & 0.000 & 0.000 & & \\
\hline & $\begin{array}{l}\text { 2nd viial } \\
\text { 3rd viial } \mathrm{c}\end{array}$ & $\begin{array}{l}\text { :oefficient, G (T) } \\
\text { oefficient, G (T) }\end{array}$ & & 1 & 1 & 0.000 & 0.000 & 0.000 & & \\
\hline & $\begin{array}{l}\text { Enthalpy } \\
\text { Hest cap } \\
\text { Hest cap }\end{array}$ & $\begin{array}{l}\text { f vaporization or sublimation, } \\
\text { city at saturation pressure, LI } \\
\text { city at constant pressure, L I }\end{array}$ & & & & & & & & \\
\hline & $\begin{array}{l}\text { Heat cap } \\
\text { Heat cap }\end{array}$ & $\begin{array}{l}\text { cily at constant pressure, L (1) } \\
\text { cily at constant pressure, G [ }\end{array}$ & & & & & & & & \\
\hline & Adiabatic & compressibility, L (T, P) & & & & & & & & \\
\hline & Speed of & ound, L (T, P] & & & & & & & & \\
\hline & alculated with & EOS & & & & & & & & \\
\hline \multirow{2}{*}{\multicolumn{3}{|c|}{ 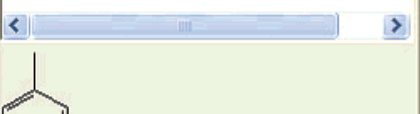 }} & & & & & & & & \\
\hline & & & & & \multicolumn{2}{|c|}{ Simplex fiting method $=$} & Refresh & Fit & Close & Help \\
\hline & & & & & & & & & & J \\
\hline & & & & & & & & & & \\
\hline
\end{tabular}

Figure 2. The EOS menu (expanded) and the EOS Control Center interface within the TDE software.

and calculation of property values. Access is provided through the EOS menu shown expanded in Figure 2. The first two menu items allow display of a REFPROP ${ }^{9}$ EOS (if available) or a previously fitted Span-Wagner EOS read from a file. (A REFPROP EOS is a reference standard EOS for a particular compound distributed with NIST Standard Reference Database 23, version 7. There are EOS representations for 48 compounds in version 7.) These are "read-only" 
in the sense that properties derived with the EOS can be displayed and compared with available experimental data, but no refitting or other modifications can be made. Selection of an EOS that is not indicated as read-only initializes the EOS fitting process for the specific equation and opens the EOS Control Center screen, shown at the right in Figure 2. The menu item Open EOS (for Fitting) opens (from a file) a Span-Wagner type EOS as an initial condition for further fitting. When opened, the EOS is scaled with the critical temperature and critical density for the compound of interest.

Depending on the selected EOS, the TDE interface provides either basic support or full support. Basic support is provided for read-only equations and includes plotting of EOS curves against experimental data and calculation of property values. Full support further includes fitting of the EOS to experimental data, calculation of covariance and uncertainties, and saving/opening Span-Wagner EOS files. Uncertainties are not calculated for the volume-translated equations, which do not provide the precision of data representation possible with the Span-Wagner EOS.

Fitting an EOS is initiated and monitored through the EOS Control Center (Figure 2). The EOS Control Center shows all properties supported by the EOS and allows full access to the data, including editing, plotting, adjustment of statistical weights for EOS fitting, and selection of the fitting method (Simplex or Simulated Annealing). Adjustment of statistical weights is done directly on the EOS Control Center form through modification of the Weight Factor (column 2) for a particular property. Only the number of data points (column 3) is shown explicitly in the form, but complete access to the underlying numerical experimental and predicted data, including bibliographic sources, is available through interface commands similar to those described previously. ${ }^{1}$ The Relative Weight (column 4) is a function that represents the relative importance of the property in the fitting process and is calculated as the sum of the squared property values multiplied by their statistical weights (as determined by TDE). The statistical weights are the reciprocal squares of the estimated uncertainties for the predicted and experimental property data. Any change by the user to the Weight Factor is directly reflected here. The Starting Error (column 5) is the error function for the property before fitting and is calculated as the sum of the squared deviations of the calculated values (with the EOS) from the experimental and predicted data multiplied by their statistical weights. The Current Error (column 6) is the error function for the property after the last completed fitting cycle.

The final column of the EOS Control Center (column 7; Adequacy) is a special function that indicates the accuracy of representation for each property. This quantity is regularly recalculated and displayed during the fitting process. By monitoring this quantity, the user can decide to interrupt a long calculation, if the results are adequate for a particular purpose. The adequacy function is defined as

$$
A=(1 / n) \Sigma\left(d_{i} / u_{i}\right)^{2}
$$

where $n$ is the number of data points, $d_{i}$ is the curve deviation of the $i$ th data point, and $u_{i}$ is the uncertainty associated with it. Achieving an adequacy $A$ value near or less than 1 indicates good property representation and is the goal of the fitting process. To ease monitoring by the user, the property rows are displayed in colors that are changed during the fit based on the value of the adequacy $A$. Fitting times for complex equations can be long, and the user can interrupt the fit at any time to review the results achieved.

Fitting of an EOS is complex and involves minimization of a multiproperty objective function, where the relative importance of properties is controlled by weighting factors for each property multiplied by data point weights, when calculating the objective function. Properties can also be excluded from fitting and calculation of the objective function based on information maintained within the EOS class.

In the case of the Span-Wagner EOS, fitting of the properties alone does not ensure that the resulting EOS will have a shape that is thermodynamically valid. Consequently, in addition to the fitting of properties, fitting constraints were added that contribute to the objective function as properties but represent deviations from certain conditions of validity for property representation. The five constraints used in TDE version 2.0 are listed at the bottom of the EOS Control Center property list (Figure 2); (1) critical temperature constraint, (2) critical density constraint, (3) critical isotherm constraint, (4) second virial constraint, and (5) heat capacity $C_{\text {sat,m }}$ (liquid) constraint.

The critical temperature $T_{\mathrm{c}}$ and critical density $\rho_{\mathrm{c}}$ constraints are used to ensure that differences between the EOS fitting parameters (nominally $T_{\mathrm{c}}$ and $\rho_{\mathrm{c}}$ ) and the $T_{\mathrm{c}}$ and $\rho_{\mathrm{c}}$ values derived in the preliminary dynamic critical evaluation are in accord with the uncertainties from the evaluation. This is analogous to the usual EOS fitting procedure in which these properties are used as fixed parameters.

The critical isotherm constraint helps to ensure that only one inflection point occurs in the curve for the critical isotherm. Figure 3 shows a valid shape for the critical isotherm of a compound with a critical density $\rho_{\mathrm{c}}$ near 300 $\mathrm{kg} \cdot \mathrm{m}^{-3}$. The final two constraints concern the shapes of the second virial coefficient $B$ variance with temperature (Figure 4) and for the $C_{\mathrm{sat}, \mathrm{m}}$ (liquid) variance with temperature (Figure 5 ). The first derivative with respect to temperature is enforced to be positive for $B$, and the second derivative with respect to temperature is enforced to be positive for $C_{\text {sat }}$ (liquid). This latter constraint is not valid for highly associating liquids, such as alcohols or acids.

The fitting methods used in TDE, involving the combination of experimental data, predicted data, and fitting constraints, allow building Span-Wagner type equations of state even for compounds with little experimental data. Such equations had previously been impossible to derive without this comprehensive approach. As with all other properties, the user can increase or decrease the relative weights of the fitting constraints and has the option to include or exclude them from fitting. Based on the selected EOS, data availability, and the known behavior of the selected EOS, TDE preselects properties and constraints as an initial guide for the user.

Fitting Span-Wagner equations of state is a complex task and may require user intervention to achieve optimum results. User actions may include changing relative weights for properties and fitting constraints, careful reviewing of experimental and predicted data with explicit rejection of suspicious data, temporary exclusion of some properties during a preliminary fit, altering the fitting method (Simplex or Simulated annealing), and starting from a successful 


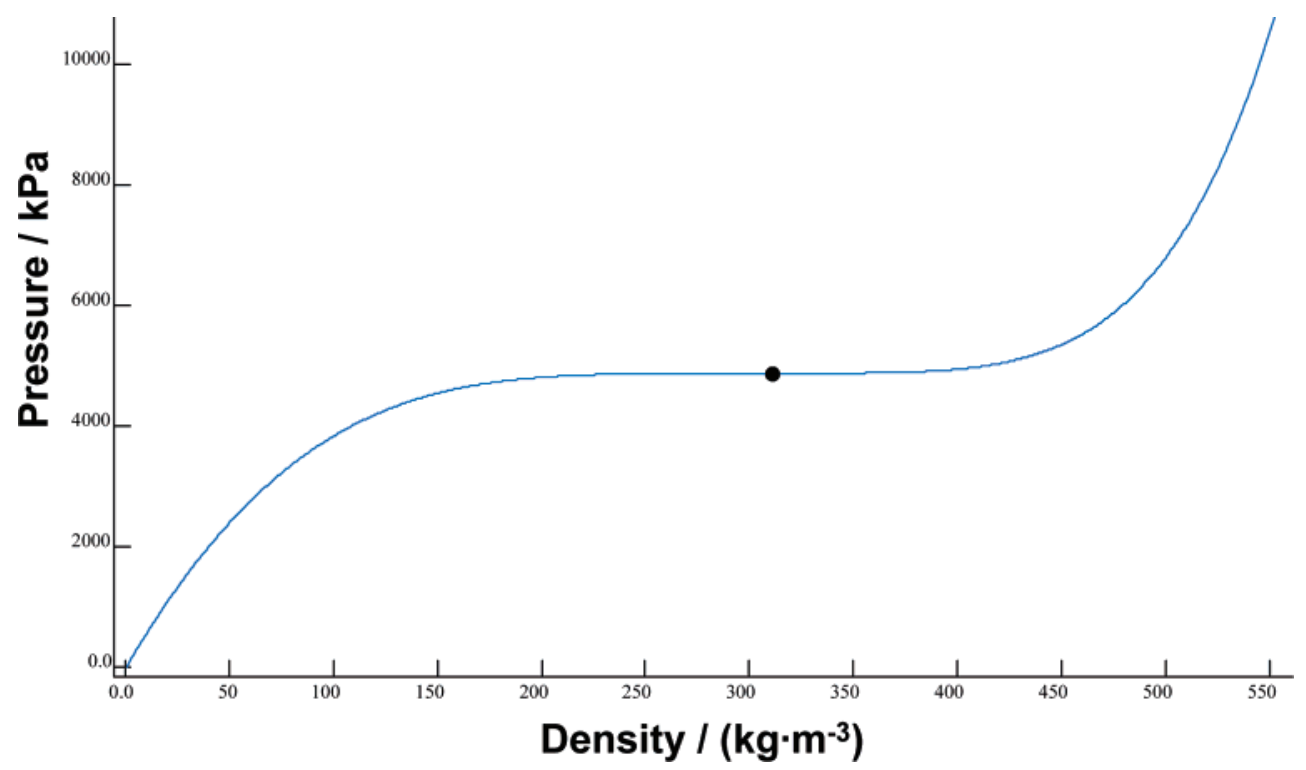

Figure 3. The line represents a valid shape for the critical isotherm showing a single inflection point at the critical density $\rho_{\mathrm{c}}$, indicated by 0 . The curve shown is for benzene.

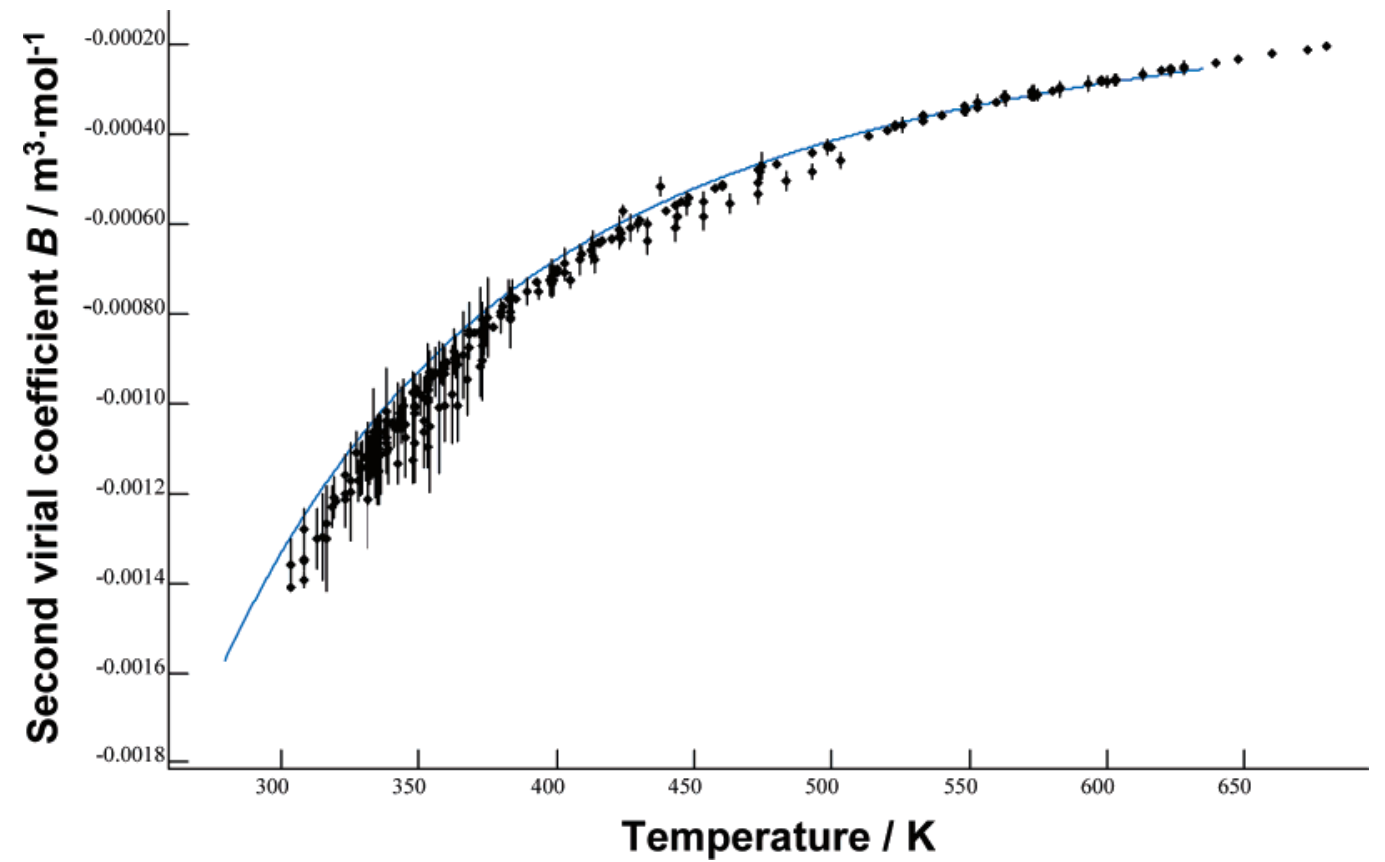

Figure 4. The curve represents a valid shape for the curve of a second virial coefficient against temperature. The data points shown are experimental values for benzene with their uncertainties.

equation (opened through the EOS menu, described earlier) for another related compound as an initial approximation. Criteria of fully successful fitting are low adequacy values (close to or less than 1) for all properties, satisfied fitting constraints (zero contributions to the objective function), valid shape of curves, and uniform scattering of experimental data along curves, as checked visually.

\section{UNCERTAINTY ESTIMATES}

Uncertainty estimates are based on the covariance approach. The standard uncertainty $u$ is calculated with the equation

$$
u^{2}=\sum_{\mathrm{i}=1}^{n} \sum_{\mathrm{j}=1}^{n} C_{\mathrm{ij}}\left(\partial \boldsymbol{P} / \partial \phi_{i}\right)\left(\partial \boldsymbol{P} / \partial \phi_{j}\right)
$$

where $C_{i j}$ is a covariance matrix element, $n$ is the number of equation parameters, and $\partial P / \partial \phi_{i}$ is the derivative of property $P$ with respect to parameter $p_{i}$. The covariance matrix is evaluated like that for any nonlinear function by assuming short-range linear dependence of the properties on the parameters. ${ }^{28}$ It is the matrix reciprocal to the matrix comprised of the elements

$$
M_{i j}=N^{-1} \sum_{k=1}^{N} w_{\mathrm{k}}\left(\partial \boldsymbol{P} / \partial \phi_{i}\right)\left(\partial \boldsymbol{P} / \partial \phi_{j}\right)
$$

where $N$ is the number of data points, $w_{k}$ is the statistical weight of the $k$ th data point, and $\left(\partial P / \partial \phi_{i}\right)_{k}$ is the derivative of the property with respect to the $i$ th parameter for the $k$ th data point for all properties used for EOS fitting. Indices $i$ and $j$ vary from 1 to the number of adjustable parameters in 


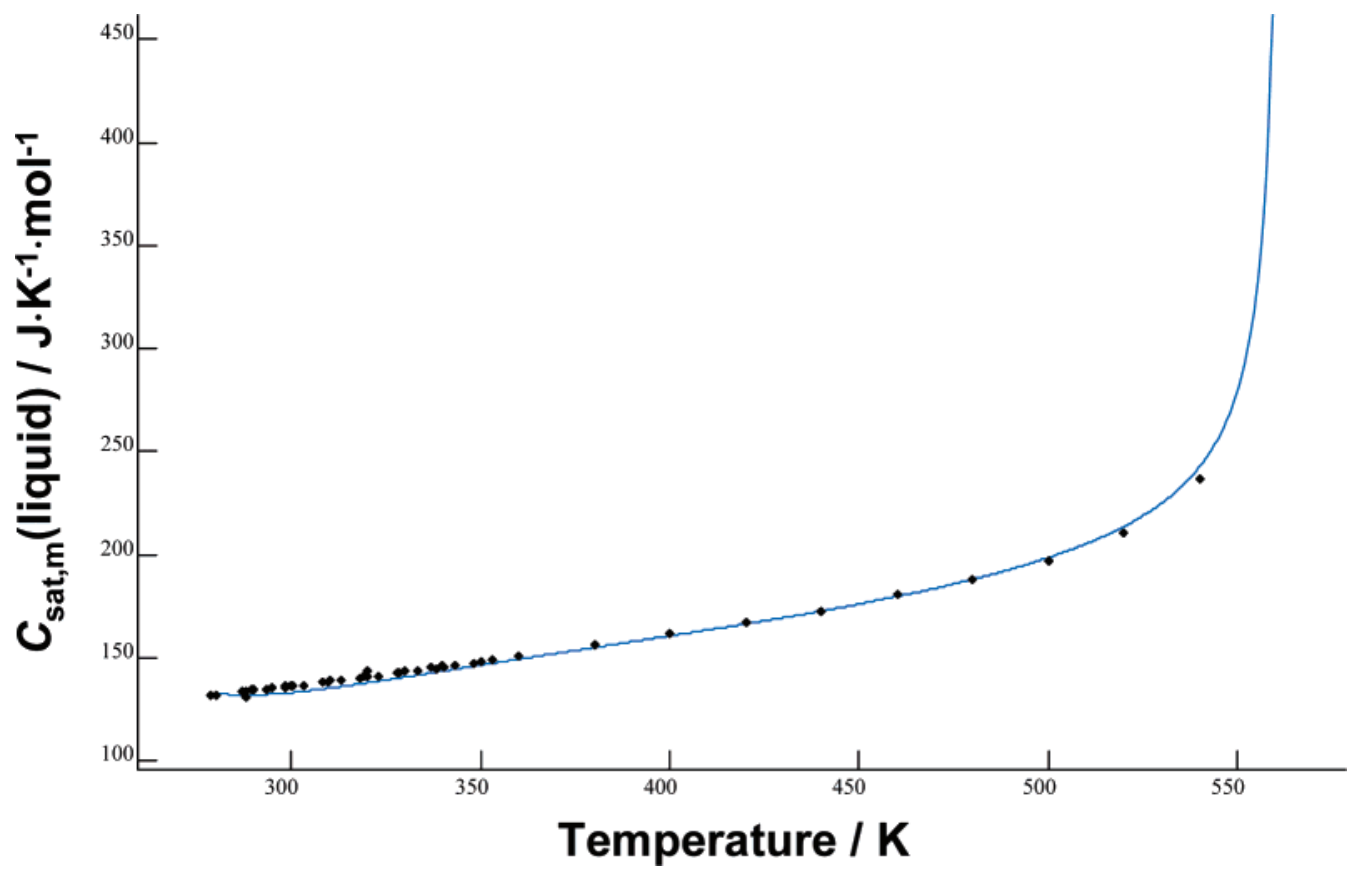

Figure 5. The curve represents a valid shape for the saturated liquid heat capacity against temperature curve. The data points shown are experimental values for benzene with their uncertainties. The sharp rise in $C_{\mathrm{sat}, \mathrm{m}}$ occurs as the critical temperature $T_{\mathrm{c}}$ is approached.

the EOS. As was described previously for $\mathrm{TDE},{ }^{1}$ both experimental uncertainties and curve deviations are taken into account when calculating statistical weights $w$ for the covariance matrix evaluation

$$
w=1 / d^{2}
$$

where $d$ is the greater of the experimental uncertainty or the curve deviation. Covariance determinations for equations of state are complicated by the fact that most properties are calculated with complex algorithms. Consequently, derivatives of these properties can be evaluated only numerically.

Equations of state such as the Span-Wagner EOS are multiparameter equations (12 parameters in TDE but as high as 56 parameters for water ${ }^{29}$ ), and the usual covariance approach for them is often inadequate, resulting in unrealistic uncertainties that are too large in regions sparsely populated with data and too small in regions of high data density. This problem has been mentioned previously by Whiting et al. ${ }^{30}$ An iterative refinement procedure was developed for such multiparameter equations in order to overcome this shortcoming for uncertainty calculations. After evaluation of the covariance matrix, uncertainties are calculated for all data points used in the fit. An "inadequacy" function $f$ is evaluated for the covariance by summation over all property data points

$$
f=N^{-1} \Sigma\left|\ln \left(u_{i} / d_{i}\right)\right|
$$

where $N$ is the number of data points, $u$ is the calculated uncertainty, $d$ is defined above, and the summation is over all data points from $i=1$ to $N$. If the inadequacy exceeds a certain value (1/8 in TDE) and the maximum number of allowed iterations ( 8 in TDE) is not exceeded, the statistical weights $w$ used for the covariance calculation are adjusted with the relation

$$
w_{\text {new }}=w_{\text {old }}(u / d)
$$

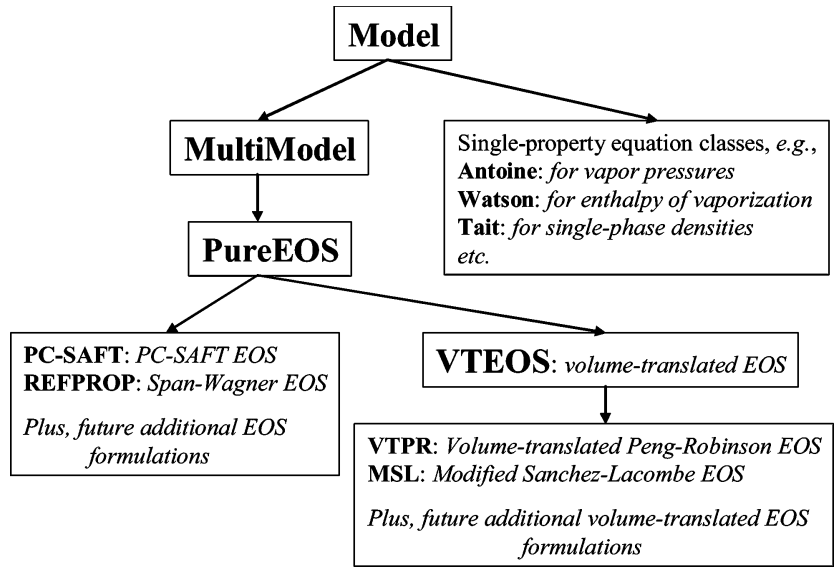

Figure 6. Hierarchy of classes (shown in boldface type) used for implementation of equations of state as property representations in TDE. The text is provided to elucidate the abbreviations used as class names.

and the covariance evaluation is repeated. The value $1 / 8$ for the inadequacy function was determined empirically.

\section{SOFTWARE ARCHITECTURE}

The software architecture for TDE was described in the first paper of this series. ${ }^{1}$ In this section, specific additions to accommodate EOS support are described, including specific $\mathrm{C}++$ classes.

The hierarchy of classes supporting equations of state (Figure 6) consists of the Model, MultiModel, PureEOS, and VTEOS classes. These define common features (data and methods) of the following objects: equation fitting for a single property (Model), equation fitting for representation of multiple properties based on a single equation (MultiModel), fitting an EOS for a pure compound (PureEOS), and fitting a volume-translated EOS for a pure compound (VTEOS). 


\section{Compound}

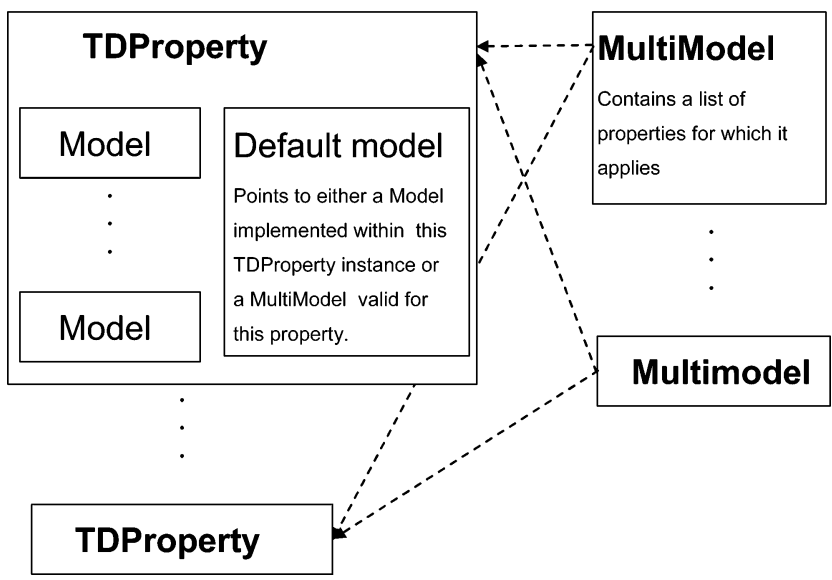

Figure 7. Structure of the Compound class in TDE.

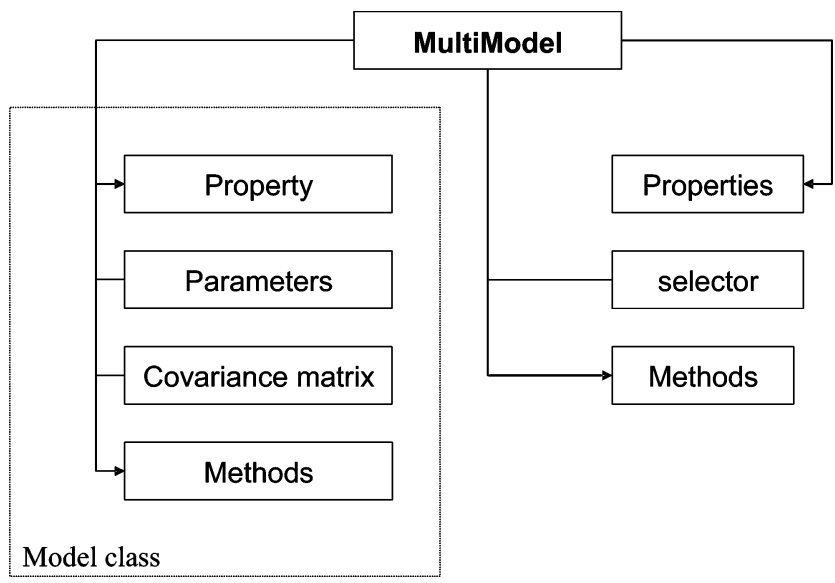

Figure 8. Structure of the MultiModel class in TDE.

The Model class stores equation parameters and provides methods for calculation of a single property together with its derivatives with respect to state variables and parameters. This class also provides methods for single property fitting and other actions. The structure of the Compound class (without the MultiModel class) was described previously in the first paper of this series, ${ }^{1}$ and its enhanced structure (with EOS support) is shown in Figure 7. The Compound class generally contains multiple TDProperty and MultiModel classes. Each TDProperty instance may use as a default Model either an internally implemented Model that is specific to that TDProperty or a MultiModel (such as an EOS) that represents multiple properties, as indicated by the dashed lines connecting various MultiModel and TDProperty classes in Figure 7. Because they are valid for multiple properties, MultiModels are implemented at the Compound level.

The structure of the MultiModel class is shown in Figure 8. The "selector" in the MultiModel class is used to specify a property for which the Model applies. The selector can be set or passed dynamically as part of a property calculation request. The MultiModel class includes methods for equation fitting, rejection of data that are of poor quality or erroneous, and covariance calculation.

While fitting and covariance calculation methods in MultiModel differ from those of Model only because of the multiple properties involved, data rejection is a very different task and requires a special solution. In order to reject bad data across multiple properties, it is necessary to compare curve deviations for different properties. For that purpose a covariance-based rejection method was developed. The covariance-based uncertainty is calculated for each data point, and then relative curve deviations are calculated as ratios of curve deviations to EOS uncertainties. The relative deviations can then be directly compared for different properties. The relative deviations also account for data density and local data quality because regions of state variables poorly populated with data are associated with higher uncertainties for calculated values.

The PureEOS class provides a property-specification function (a method for setting the "selector" of the MultiModel class) based on the properties, phases, and state variables involved and a function for calculation of all supported properties based on the excess Helmholtz energy and its derivatives for the EOS instance. Properties are calculated as functions of temperature and density based on published thermodynamic relationships. ${ }^{16}$ Special functions calculate (a) density as a function of temperature and pressure, (b) the saturated pressures and densities as functions of temperature, and (c) the coordinates of the critical point, i.e., where saturated liquid and gas densities converge. Instances of PureEOS provide functions for calculation of the excess Helmholtz energy based on a specific equation of state. Ideal-gas heat capacities as functions of temperature are needed by each EOS, and these are provided in TDE through an auxiliary equation.

As shown in the class hierarchy (Figure 6), PC-SAFT and REFPROP classes are directly derived from the PureEOS class. The PC-SAFT class implements all functions necessary for calculation of $a^{r}$ and its derivatives for a PC-SAFT EOS. The REFPROP class uses the REFPROP (NIST SRD 23) ${ }^{9}$ dynamic link library as the source of read-only EOS parameters for compounds included in that database. The REFPROP dynamic link library is also used as the engine for performing property calculations with Span-Wagner type equations of state. ${ }^{16}$

The VTEOS class applies the volume-translation term for the calculation of single-phase and saturated densities of the liquid and gas, as described above, after an EOS-based calculation for these properties. The volume-translated Peng-Robinson (VTPR) and modified Sanchez-Lacombe (MSL) classes are derived from the VTEOS class (Figure 6).

\section{DYNAMIC UPDATES OVER THE WEB}

An essential aspect of dynamic data evaluation is access by the user to a near comprehensive collection of experimental data, including the most recent data from the literature. As previously described, ${ }^{1}$ experimental data used by TDE (version 1.0) were extracted from the NIST/TRC SOURCE $^{17}$ system and distributed as a local database (TDE SOURCE) with the program. NIST/TRC SOURCE is maintained at NIST and is continuously populated with new experimental data (using Guided Data Capture GDC software $^{31}$ developed at NIST). In order to more closely adhere to the requirements for dynamic data evaluation, TDE (version 2.0) includes a mechanism for dynamically updating the local TDE SOURCE based on additions and changes to NIST/TRC SOURCE through a central server. 


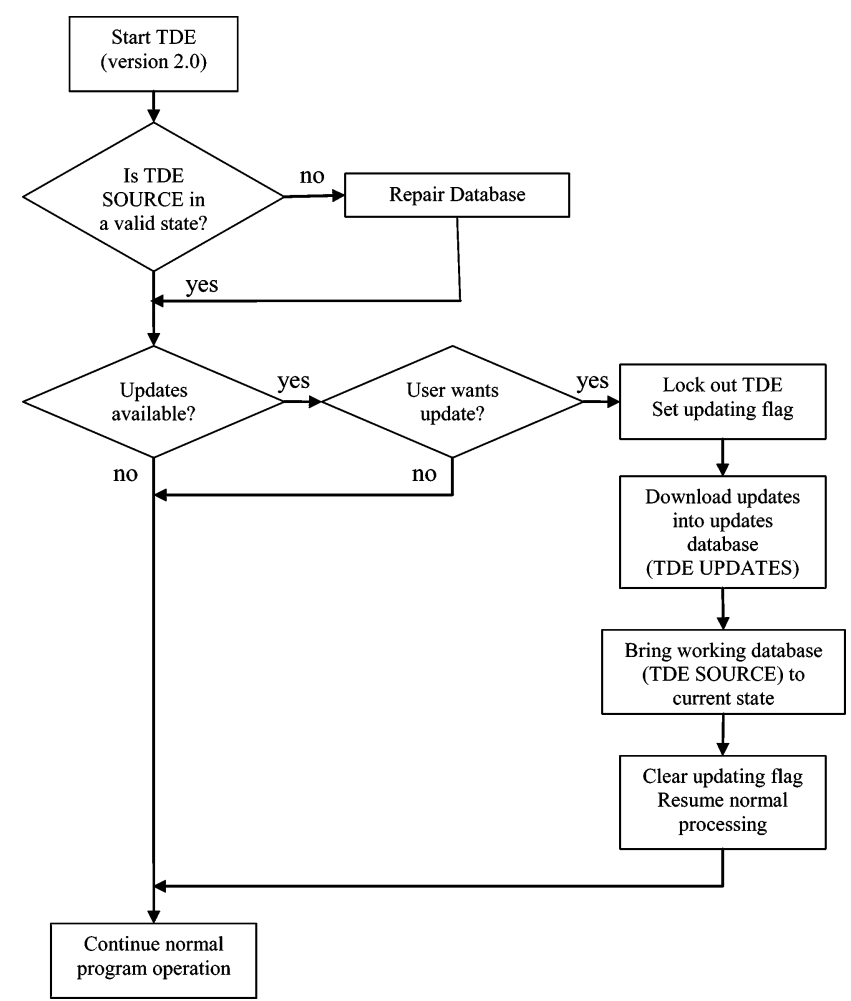

Figure 9. Flow chart for dynamic updating of the TDE SOURCE database from the Web through the local TDE UPDATES database.

Multiple technical approaches were considered for this update procedure, and a key decision was to provide periodic (quarterly) database updates rather than allowing continuous access to NIST/TRC SOURCE. This reduces the variety of possible TDE SOURCE data conditions to a small number of well defined states associated with particular dates and allows for unequivocal evaluation traceability, a key requirement for many engineering applications. A second decision was to maintain a local database of differences (TDE UPDATES) that allows each instance of TDE to set or restore the working database (TDE SOURCE) to any of the allowed discrete states rather than maintaining traceability information for every individual data point.

The process by which TDE checks for updates and maintains current TDE SOURCE information is shown as a flow chart in Figure 9. When started, TDE launches a background process that checks for the availability of a new update. If available, TDE prompts the user to download and install the update.

Once the user initiates the update process, the current update file is downloaded and merged with the local updates database (TDE UPDATES). TDE SOURCE is then set to the most recent valid state or version. Each update contains both records to be removed and records to add. When the TDE SOURCE state is changed (i.e., when the user selects an alternative TDE SOURCE version through a Set database version command in the Updates menu of the main toolbar), the change occurs in a stepwise fashion to neighboring states until the target state is reached, thus allowing any historical state of the database to be reproduced. The database version is stored along with evaluation results as an output of TDE, allowing for complete reproducibility and traceability for any evaluation. Setting the TDE SOURCE database to a given version and doing an evaluation with a given version of TDE will always give the same results.

A special variable is set when the TDE SOURCE version change procedure is started and cleared when finished. This allows database integrity to be maintained and avoids the problem of TDE evaluations based on an indefinite database state. One example of an occurrence that would cause the TDE SOURCE state to be indefinite is an unexpected shutdown of the user's computer system during download of updates. When TDE is launched, the TDE SOURCE integrity (or "validity") is checked, and any database repairs are performed before any other functions are activated, as shown in the first step of the flow chart (Figure 9). Furthermore, when doing any state-change or repair operation, TDE does not allow the user to exit from the program.

Details of Implementation. The list of valid TDE SOURCE states is maintained as an index file in a coded list on the central server at NIST. The index also contains link information to the encrypted update files on the server. Each update file contains all the information necessary for transition from the preceding TDE SOURCE state to a new state. This information consists of a complete list of records to delete and new records to add. For instance, if a record has been changed since the preceding version, two records appear in the update file: a record to delete and a record to add.

To produce the difference information for updating TDE SOURCE to the current state of NIST/TRC SOURCE, software was developed that does two series of comparisons and saves two kinds of records: records present in TDE SOURCE but absent in NIST/TRC SOURCE and records present in NIST/TRC SOURCE but absent in TDE SOURCE. The first group is the set of records to delete, and the second group is the set of records to add.

\section{COMPARISONS WITH PROPERTY-BY-PROPERTY AND LITERATURE EOS EVALUATIONS}

As described in the first paper of this series, version 1.0 of TDE used separate equations for each evaluated property, with specific thermodynamic relationships between properties subsequently applied as constraints. As described here, version 2.0 includes this capability but further provides for deployment of equations of state that are inherently thermodynamically consistent (excluding volume-translated equations). In version 1.0, gas-phase densities were represented with the virial equation truncated at the third virial coefficient. Deployment of EOS technology in TDE version 2.0 marks a major extension into the supercritical region for property evaluations.

Span-Wagner EOS representations made with TDE for compounds included in the REFPROP database ${ }^{9}$ are nearly indistinguishable from those published in REFPROP. This is due, in part, to the use in TDE of the REFPROP parameters as starting conditions. Some unexpected results are displayed in Figure 10, which shows deviation plots for vapor pressures of cyclohexane derived with an EOS formulated by Span and Wagner ${ }^{16 \mathrm{~b}}$ (upper figure) and by TDE (lower figure). In the initial EOS fits published with the equation development, Span and Wagner noted some apparent inconsistencies in the available experimental data and recommended additional measurements to resolve the discrepancies. For this com- 

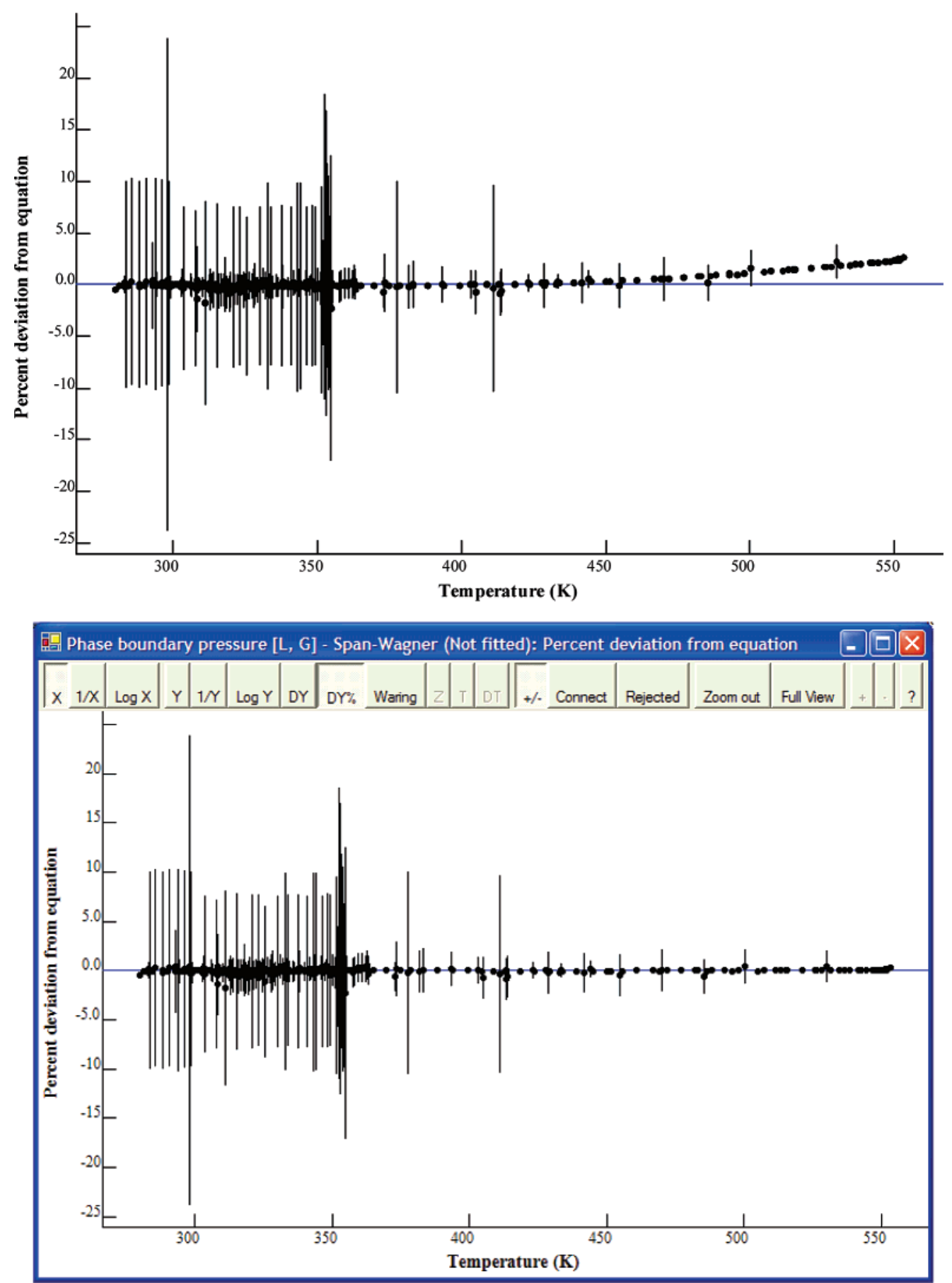

Figure 10. Deviation plots for experimental vapor pressures of cyclohexane relative to an EOS reported in the literature ${ }^{16 \mathrm{~b}}$ (upper figure) and evaluated with TDE (lower figure).

pound, the TDE algorithm provided a successful representation of the experimental vapor pressures, while maintaining a high-quality fit for densities and heat capacities.

As a further test of the capabilities of TDE, Span-Wagner EOS representations for benzene were generated with two very different data scenarios: (1) with saturation line (vapor pressure and density) data, plus single phase densities, and (2) with saturation line data only. The results showed that in the region more than $10 \mathrm{~K}$ removed from $T_{\mathrm{c}}$ the singlephase densities calculated with the Span-Wagner EOS generated by TDE on the basis of the saturation line data only were within $4 \%$ of the experimental single-phase data for benzene. Though outside the experimental uncertainties, such a result is excellent, considering the limited data set used.

\section{DYNAMICALLY LINKED LIBRARY (DLL) VERSION OF TDE}

A DLL implementation of TDE 2.0 has been developed to provide convenient mechanisms for implementation of the TDE technology into chemical engineering software applications. This version supports all aspects of property-byproperty data evaluation for processing of one chemical compound at a time. All supported alternative equations for a given property are available through the DLL, and the results are returned in ThermoML ${ }^{6}$ format. 
Table 2. Equation Parameters for the PC-SAFT Equation of State Required for Full Specification of the EOS Formulation ${ }^{a}$

\begin{tabular}{rcccrrr}
\hline$i$ & $a_{0}$ & $a_{1}$ & $a_{2}$ & \multicolumn{1}{c}{$b_{0}$} & $b_{1}$ \\
\hline 0 & 0.910563145 & -0.308401692 & -0.090614835 & 0.724094694 & -0.575549808 & 0.097688312 \\
1 & 0.636128145 & 0.186053116 & 0.452784281 & 2.238279186 & 0.699509552 & -0.255757498 \\
2 & 2.686134789 & -2.503004726 & 0.596270073 & -4.002584948 & 3.892567339 & -9.155856153 \\
3 & -26.54736249 & 21.41979363 & -1.724182913 & -21.00357681 & -17.21547165 & 20.64207597 \\
4 & 97.75920878 & -65.25588533 & -4.130211253 & 26.85564136 & 192.6722645 & -38.80443005 \\
5 & -159.5915409 & 83.31868048 & 13.77663187 & 206.5513384 & -161.8264616 & 93.62677408 \\
6 & 91.29777408 & -33.74692293 & -8.672847037 & -355.6023561 & -165.2076935 & -29.66690559
\end{tabular}

${ }^{a}$ The parameters are used in eqs A14 and A15.

\section{CONCLUSIONS AND FUTURE DEVELOPMENT}

(1) The expansion of the software implementation of the dynamic data evaluation concept within the NIST ThermoData Engine described in the present paper provides unique opportunities for generation of equations of state on-demand based on specific data scenarios. This is a new principal capability that enables critically evaluated thermodynamic property data to be used in a broad range of engineering applications and over an extensive temperature-pressuredensity variable space.

(2) A new Web-based technology that provides updates for the TDE-SOURCE data storage facility used in conjunction with the critical data evaluation within the NIST ThermoData Engine makes the overall process fully dynamic.

(3) The algorithms and software tools developed for generation of the equations of state on-demand and Webbased technology for TDE-SOURCE dynamic updates complete the implementation of the dynamic data evaluation concept for pure chemical species.

(4) A DLL version of TDE makes it convenient to incorporate the TDE technology into chemical engineering software applications.

Further development will include expansion of TDE to more complex systems, such as binary mixtures and incorporation of predictive methods beyond group contribution and corresponding states techniques. Additional EOS formulations will be added to TDE based on user demand.

\section{ACKNOWLEDGMENT}

The authors express their appreciation to Drs. S. Watanasiri (AspenTech, Cambridge, MA), M. Satyro (Virtual Materials Group, Calgary, Canada), and A. I. Johns (National Engineering Laboratory, Glasgow, U.K.) for their very valuable advice during development of TDE. In addition, the authors thank Drs. A. H. Harvey, M. L. Huber (both of NIST, Boulder, CO) and Dr. F. J. Doyle (Frank J. Doyle Enterprises, Woodlands, TX) for their practical suggestions during testing of TDE. Finally, the authors wish to acknowledge the late Dr. Randolph Wilhoit of Texas A \& M University, who was an inspiration for the implementation of the dynamic data evaluation concept. This is a contribution of the National Institute of Standards and Technology, not subject to copyright in the United States. Trade names provided are only to specify procedures adequately and do not imply endorsement by the National Institute of Standards and Technology. Similar products by other manufacturers may be found to work as well or better.

\section{APPENDIX: MATHEMATICAL FORMULATION OF THE PC-SAFT EQUATION OF STATE IN TDE}

The PC-SAFT EOS is implemented in the formulation described by Gross and Sadowski, ${ }^{12}$ where the primary quantity calculated is the dimensionless residual Helmholtz energy $a^{r}$. The quantity $a^{r}$ is calculated as follows, with $m$, $\sigma$, and $\epsilon$ as equation parameters and $a$ and $b$ as arrays of constants. Aside from the molar density $\rho$ and temperature $T$, all other quantities are intermediate to simplify writing of the equation. Generally, symbols used are those of Gross and Sadowski. ${ }^{12}$ The dimensionless residual Helmholtz energy $a^{r}$ is

$$
a^{r}=\left(A-A^{0}\right) /(R \cdot T)
$$

where $A$ is the Helmholtz energy for the real-fluid, $A^{0}$ is the ideal-gas Helmholtz energy at the same temperature and molar density, and $\rho$ is the molar density. This quantity is expressed as the sum

$$
a^{r}=\mathrm{a}^{\mathrm{h}}+\mathrm{a}^{\mathrm{d}}
$$

The quantity $\mathrm{a}^{\mathrm{h}}$ is defined by the following expressions

$$
\mathrm{a}^{\mathrm{h}}=m \cdot a^{\phi}-\{(m-1) \cdot \ln (g)\}
$$

where

$$
\begin{gathered}
a^{\phi}=\left[3 \xi(1) \xi(2) /\{1-\xi(3)\}+\xi(2)^{3} /\left(\xi(3)\{1-\xi(3)\}^{2}\right)+\right. \\
\left.\left\{\xi(2)^{3} / \xi(3)^{2}-\xi(0)\right\} \ln \{1-\xi(3)\}\right] / \xi(0) \text { (A4) } \\
g=1 /\{1-\xi(3)\}+3 \cdot \delta \cdot \xi(2) /\left[2 \cdot\{1-\xi(3)\}^{2}\right]+\delta^{2} \cdot \xi(2)^{2} / \\
{\left[2 \cdot\{1-\xi(3)\}^{3}\right](\mathrm{A} 5)} \\
\xi(\mathrm{n})=(\pi / 6) \rho m \delta^{\mathrm{n}} \\
\delta=\sigma\left(1-0.12 \mathrm{e}^{-3 \epsilon / T}\right)
\end{gathered}
$$

The quantity $\mathrm{a}^{\mathrm{d}}$ is defined by the following expressions

$$
\mathrm{a}^{\mathrm{d}}=-\pi \rho\left(2 I_{1} e_{1}+m C_{1} I_{2} e_{2}\right)
$$

where

$$
\begin{gathered}
I_{1}=\sum_{i=0}^{6} a_{i} v^{i} \quad(a \text { and } v \text { are defined below }) \\
e_{1}=m^{2} \epsilon / T \sigma^{3}
\end{gathered}
$$




$$
\begin{gathered}
C_{1}=1 /\left[1+m\left(8 v-2 v^{2}\right) /(1-v)^{4}+(1-m)(20 v-\right. \\
\left.\left.27 v^{2}+12 v^{3}-2 v^{4}\right) /\{(1-v)(2-v)\}^{2}\right] \quad(\mathrm{A} 11) \\
I_{2}=\sum_{i=0}^{6} b_{i} v^{i} \quad(b \text { and } v \text { are defined below }) \\
e_{2}=m^{2}(\epsilon / T)^{2} \sigma^{3}
\end{gathered}
$$

The parameters $a, b$, and $v$ in eqs A8 and A11 are defined here with the coefficients $a_{x}(i)$ and $b_{x}(i)(i=0-6)$ given in Table 2 .

$$
\begin{gathered}
a_{i}=a_{0}(i)+\{(m-1) / m\} a_{1}(i)+\{(m-1) / m\}\{(m- \\
2) / m\} a_{2}(i)(\mathrm{A} 14) \\
b_{i}=b_{0}(i)+\{(m-1) / m\} b_{1}(i)+\{(m-1) / m\}\{(m- \\
2) / m\} b_{2}(i)(\mathrm{A} 15) \\
v=\xi(3)
\end{gathered}
$$

Initial approximations for the three adjustable parameters $m, \sigma$, and $\epsilon$ are based on the critical temperature $T_{\mathrm{c}}$ and the molar mass $M$ expressed in $\mathrm{K}$ and $\mathrm{g} \cdot \mathrm{mol}^{-1}$, respectively

$$
\begin{gathered}
m=0.833+0.0000333 M T_{\mathrm{c}} \\
\sigma=3.8 \\
\epsilon=66.7+0.333 T_{\mathrm{c}}
\end{gathered}
$$

\section{REFERENCES AND NOTES}

(1) Frenkel, M.; Chirico, R. D.; Diky, V.; Yan, X.; Dong, Q.; Muzny, C. ThermoData Engine (TDE): Software Implementation of the Dynamic Data Evaluation Concept. J. Chem. Inf. Model. 2005, 45, 816-838.

(2) Frenkel, M.; Chirico, R. D.; Diky, V. V.; Yan, X.; Dong, Q.; Muzny, C. NIST ThermoData Engine, NIST Standard Reference Database 103 , Version 1.0; National Institute of Standards and Technology, Standard Reference Data Program: Gaithersburg, MD, 2004.

(3) (a) Wilhoit, R. C.; Marsh, K. N. Future Directions for Data Compilation. Int. J. Thermophys. 1999, 20, 247-255. (b) Frenkel, M. Dynamic Compilation: A Key Concept for Future Thermophysical Data Evaluation. In Forum 2000: Fluid Properties for New Technologies - Connecting Virtual Design with Physical Reality; Rainwater, J. C., Friend, D. G., Hanley, H. J. M., Harvey, A. H., Holcomb, C. D., Laesecke, A., Magee, J. W., Muzny, C., Eds.; NIST Special Publication 975, Gaithersburg, MD, 2001; pp 83-84.

(4) Frenkel, M. Global Communications and Expert Systems in Thermodynamics: Connecting Property Measurement and Chemical Process Design. Pure Appl. Chem. 2005, 77, 1349-1367.

(5) Frenkel, M.; Chirico, R. D.; Diky, V. V.; Marsh, K. N.; Dymond, J. H.; Wakeham, W. A. ThermoML - An XML-Based Approach for Storage and Exchange of Experimental and Critically Evaluated Thermophysical and Thermochemical Property Data. 3. Critically Evaluated Data, Predicted Data, and Equation Representation. J. Chem. Eng. Data 2004, 49, 381-393.

(6) Frenkel, M.; Chirico, R. D.; Diky, V. V.; Marsh, K. N.; Dymond, J. H.; Wakeham, W. A.; Stein, S. E.; Königsberger, E.; Goodwin, A. R. H. XML-based IUPAC Standard for Experimental, Predicted, and Critically Evaluated Thermodynamic Property Data Storage and Capture (ThermoML): IUPAC Recommendations 2006. Pure Appl. Chem. 2006, 78, 541-612.

(7) Poling, B. E.; Prausnitz, J. M.; O'Connell, J. P. The Properties of Gases and Liquids, 5th ed.; McGraw Hill: New York, 2001; pp 4.84.11 .

(8) (a) NIST/TRC Thermodynamic Tables - Hydrocarbons; Frenkel, M., Ed.; NSRDS-NIST 74, National Institute of Standards and Technology, Standard Reference Data Program: Gaithersburg, MD, 2000-2006. These tables have been published continuously since 1942. (b) NIST/ TRC Thermodynamic Tables - Non-Hydrocarbons; Frenkel, M., Ed NSRDS-NIST 75, National Institute of Standards and Technology, Standard Reference Data Program: Gaithersburg, MD, 2000-2006. These tables have been published continuously since 1942
(9) Lemmon, E. W.; McLinden, M. O.; Huber, M. L. NIST Standard Reference Database 23: Reference Fluid Thermodynamic and Transport Properties-REFPROP, Version 7.0; National Institute of Standards and Technology, Standard Reference Data Program: Gaithersburg, MD, 2002.

(10) Frenkel, M.; Chirico, R. D.; Diky, V. V.; Muzny, C.; Lemmon, E. W.; Yan, X.; Dong, Q. NIST ThermoData Engine, NIST Standard Reference Database 103, Version 2.0; National Institute of Standards and Technology, Standard Reference Data Program: Gaithersburg, MD, 2006. http://www.nist.gov/srd/WebGuide/nist103/103v2.htm (accessed Apr 10, 2007)

(11) Peng, D.-Y.; Robinson, D. B. A New Two-Constant Equation of State. Ind. Eng. Chem. Fundam. 1976, 15, 59-64.

(12) Gross, J.; Sadowski, G. Perturbed-Chain SAFT: An Equation of State Based on a Perturbation Theory for Chain Molecules. Ind. Eng. Chem. Res. 2001, 40, 1244-1260.

(13) Koak, N.; Heidemann, R. A. Polymer-Solvent Phase Behavior near the Solvent Vapor Pressure. Ind. Eng. Chem. Res. 1996, 35, 43014309.

(14) Krenz, R. A. Correlating the Fluid Phase Behavior of Polydisperse Polyethylene Solutions using the Modified Sanchez-Lacombe Equation of State, Ph.D. Thesis, University of Calgary, Alberta, CA, 2005.

(15) Lemmon, E. W.; Jacobsen, R. T. A New Functional Form and New Fitting Techniques for Equations of State with Application to Pentafluoroethane (HFC-125). J. Phys. Chem. Ref. Data 2005, 34 69-108.

(16) (a) Span, R.; Wagner, W. Equations of State for Technical Applications. I. Simultaneously Optimized Functional Forms for Nonpolar and Polar Fluids. Int. J. Thermophys. 2003, 24, 1-39. (b) Span, R.; Wagner, W. Equations of State for Technical Applications. II. Results for Nonpolar Fuids. Int. J. Thermophys. 2003, 24, 41-109. (c) Span, R.; Wagner, W. Equations of State for Technical Applications. III. Results for Polar Fuids. Int. J. Thermophys. 2003, 24, 111-161.

(17) Frenkel, M.; Dong, Q.; Wilhoit, R. C.; Hall, K. R. TRC SOURCE Database: A Unique Tool for Automatic Production of Data Compilations. Int. J. Thermophys. 2001, 22, 215-226.

(18) Roy-Faderman, A.; Koletzke, P.; Dorsey, P. Oracle JDeveloper $10 \mathrm{~g}$ Handbook; McGraw-Hill: New York, 2004; pp 184-186.

(19) NIST Ionic Liquids Database, ILThermo. NIST Standard Reference Database 147; National Institute of Standards and Technology, Standard Reference Data Program: Gaithersburg, MD, 2006. http:// ilthermo.boulder.nist.gov/ILThermo/ (accessed Apr 10, 2007).

(20) Ihmels, E. C.; Lemmon, E. W. Experimental Densities, Vapor Pressures, and Critical Point, and a Fundamental Equation of State for Dimethyl Ether. Fluid Phase Equilib. 2007, in press.

(21) Lemmon, E. W.; Jacobsen, R. T. A New Functional Form and New Fitting Techniques for Equations of State with Application to Pentafluoroethane (HFC-125). J. Phys. Chem. Ref. Data 2005, 34 69-108

(22) Lemmon, E. W.; Span, R. Short Fundamental Equations of State for 20 Industrial Fluids. J. Chem. Eng. Data 2006, 51, 785-850.

(23) Magoulas, K.; Tassios, D. Thermophysical Properties of n-Alkanes from $\mathrm{C} 1$ to $\mathrm{C} 20$ and their Prediction for Higher Ones. Fluid Phase Equilib. 1990, 56, 119-140.

(24) Peneloux, A.; Rauzy, E. A consistent correction for Redlich-KwongSoave volumes. Fluid Phase Equilib. 1982, 8, 7-23.

(25) Gross, J.; Sadowski, G. Application of the Perturbed-Chain SAFT Equation of State to Associating Systems. Ind. Eng. Chem. Res. 2002, 41, 5510-5515

(26) Wolfram Research, Inc. Mathematica, Version 5.2; Champaign, IL, 2005.

(27) Frenkel, M.; Kabo, G. J.; Marsh, K. N.; Roganov, G. N.; Wilhoit, R. C. Thermodynamics of Organic Compounds in the Gas State, Volumes I and II; Thermodynamics Research Center: College Station, TX 1994.

(28) Bates, D. M.; Wats, D. G. Nonlinear Regression Analysis and Its Applications; Wiley: New York, 1988; pp 58-59.

(29) Wagner, W.; Pruss, A. The IAPWS Formulation 1995 for the Thermodynamic Properties of Ordinary Water Substance for General and Scientific Use. J. Phys. Chem. Ref. Data 2002, 31, 387-535.

(30) Whiting, W. B.; Vasquez, V. R.; Meercshaert, M. M. Techniques for assessing the effects of uncertainties in thermodynamic models and data. Fluid Phase Equilib. 1999, 158-160, 627-641.

(31) Diky, V. V.; Chirico, R. D.; Wilhoit, R. C.; Dong, Q.; Frenkel, M. Windows-Based Guided Data Capture Software for Mass-Scale Thermophysical and Thermochemical Property Data Collection. J. Chem. Inf. Comput. Sci. 2003, 43, 15-24. See http://www.trc.nist.gov/ GDC.html (accessed Apr 10, 2007).

CI700071T 